\title{
Object-Based Media: A Toolkit for Building Responsive Content.
}

\author{
Mike Armstrong \\ BBC R\&D \\ MediaCityUK Salford \\ mike.armstrong@bbc.co.uk
}

\begin{abstract}
This paper outlines the work at BBC R\&D on creating a toolkit for creating object-based media content that responds to the display device and user interaction. The aim in creating this tool kit is to create a suite of tools for producers to create responsive media without the need for bespoke computer code and data structures.
\end{abstract}

Media, narrative, responsive media, media production, media consumption, radio, television.

\section{INTRODUCTION}

Object-Based Media (OBM) is a way of making media content that can be assembled in different ways to meet differing requirements for users and their devices. This enables the sound, video, text and graphics to be tailored by the user's device from a series of component parts - see Figure 1. This process can enable the same narrative content to be consumed in different ways as well as providing alternative routes through a narrative, dependent upon the user's interests and needs (1).

We have created a number of individually crafted pieces of object-based media in the course of our research, from a short locative radio drama, to a variable length radio documentary, a cooking tutorial and most recently an origami tutorial. However, all these items have required a hand crafted approach to their creation which essentially has involved writing computer code and data structures as much, if not more than it has the process of media production (2).

TRADITIONAL MEDIA

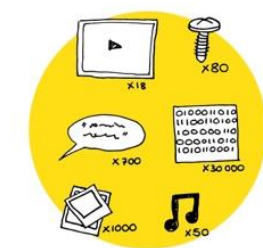

Media is captured using traditional tools.

\section{OBJECT BASED MEDIA}

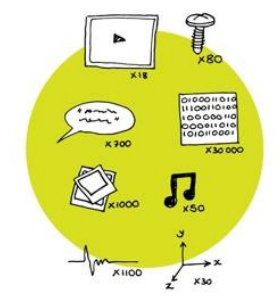

Media is captured using new and cols. Metadata is produced and recorded.
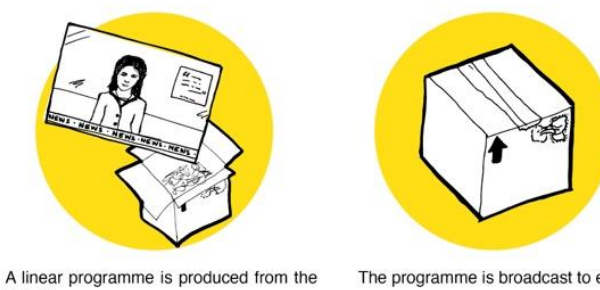

The programme is broadcast to everyone. media.

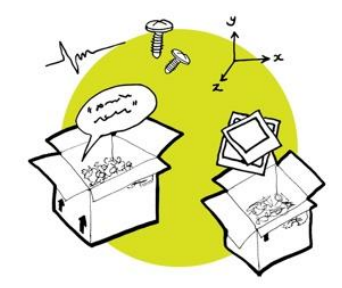

These are packaged as a collection of objects.

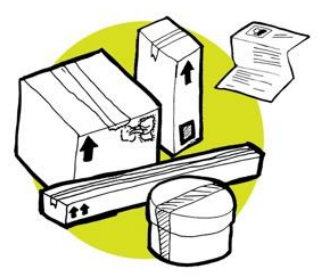

The objects are broadcast to everyone accompanied by the metadata, which can be assembled.

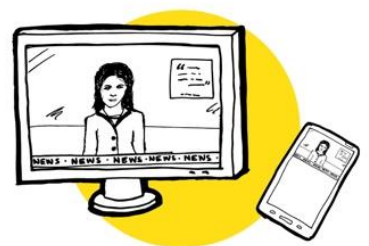

The same content is played back on all The same content is played back on all
devices, resulting in compromises for
some experiences.

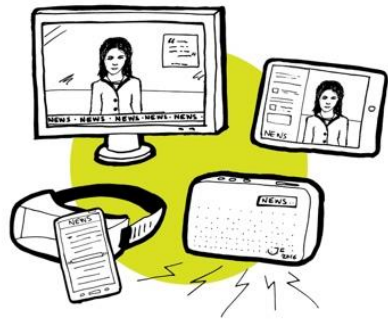

Individual devices in the home assemble objects according to the metadata, viewer in the context of their devices. environment, and preference.

BBC | R\&D

Figure 1: How object-based media contrasts with traditional A/V media. 


\section{THE OBM TOOLKIT}

The key challenge for the programme maker crafting a single finished piece of audio/visual media is expressing their editorial intentions in a form which can be interpreted to best meet the needs of the individual audience members. For example, in an audio mix, the levels of narrative speech, narrative effects, background effects and music can be altered to suite people's hearing (3), which requires data about the narrative roles of these elements.

So the tools needed for media composition now need to start by expressing the logical structure of the experience, then move on to populating that experience with media and data that can then be experienced through a player (4).

We are currently in the process of creating the three main elements of the OBM toolkit. These are evolving as we learn more about the requirements of the underlying data models for each of the types of media experience.

\subsection{Narrative structure tool}

There are two main ways in which media can be rearranged, the first is the scheduling of the media objects in a temporal order and the second is the layering and processing of objects, as with an audio mix or a graphical overlay. In more complex experiences some of the layers may be sequenced separately dependant on the user's needs.

A data model is required which enables the producer to express the narrative structure and user interaction separately from the media used to convey the narrative, particularly where there may be more than one form of media that expresses a narrative item, e.g. alternative camera views or modal substitution.

Creating user interfaces which enable the producer to clearly express their narrative ideas is a considerable challenge as it involves a change of perspective, to one that focuses on the higher-level ideas rather than the expression of those ideas directly in sound and video. This includes the design of the interaction between the user, their device and the media.

\subsection{Media capture}

Once the experience has been expressed as a narrative structure, then the capture of the media assets can then populate the data structure. Initially these could be proxy items such as still images that enable the sequencing and user interaction to be previewed which are then replaced with audio and video to create the experience.

Here the initial planning work involved in creating the experience structure should enable better tracking of rushes and versions, helping to streamline the process of production. New user interfaces are required for the ingest of logging information during capture.

\subsection{Media player}

The final piece of the toolkit is the media player itself. This must handle the media selection, scheduling and layering along with all the user and device interactions. It must combine the narrative structure and logic with the user interactions and device capabilities to render the experience in an appropriate manner. The first version of this player has been deployed successfully on BBC Taster for the MAKE! Craft Britain interactive Origami tutorial. (5)

\section{RESEARCH DIRECTION}

Our work on developing this toolkit goes hand in hand with developing our understanding of the potential of $\mathrm{OBM}$. As with $360^{\circ}$ video we have to formulate new approaches to storytelling. Key to this will be understanding how to retain the user's continual immersion in an experience whilst enabling them to interact with the content. It will also be important to understand what forms of content benefit most from an object-based approach, along with the new possibilities it brings. We are looking to work with a wide range of partners from industry and academia along with freelance producers as part of a community of practice.

\section{REFERENCES}

1) Armstrong, M., Brooks, M., Churnside, A., Evans, M., Melchior, F. Shotton, M., (2014) Object-Based Broadcasting - Curation, Responsiveness and User Experience. IBC 2014.

2) Evans, M., Ferne, T., Watson, Z., Melchior, F., Brooks, M., Stenton, P. Forrester, I., (2016) Creating Object-Based Experiences in the Real World. IBC 2016.

3) Ward, L. Shirley, B. Davies, WJ. (2017) Big Pictures and Little Screens: How television sound research can work with, and for, hard of hearing viewers. Proceedings of the Institute of Acoustics, Vol. 39. Pt.1 2017.

4) Cox, J. Brooks, M. Forrester, I. Armstrong, M. (2017) Moving Object-Based media production from one-off examples to scalable workflows. IBC 2017.

5) Cox, J. Brooks, M. (2018) How we Made the Make-Along. https://www.bbc.co.uk/rd/blog/201803-make-along-production-object-based-media (retrieved March 2018). 